\title{
Recent Efforts for Credible CFD Simulations in China
}

\author{
Li Li, Bai Wen, and Liang Yihua \\ Aeronautics Computing Technique Research Institute (ACTRI), P.O. Box 90, Xi'an 710068, China \\ Correspondence should be addressed to Li Li, westlili@163.com
}

Received 6 October 2010; Accepted 12 January 2011

Academic Editor: Guan Yeoh

Copyright () $2011 \mathrm{Li} \mathrm{Li}$ et al. This is an open access article distributed under the Creative Commons Attribution License, which permits unrestricted use, distribution, and reproduction in any medium, provided the original work is properly cited.

\begin{abstract}
In this paper some recent efforts for credible computational fluid dynamics (CFD) simulations in China are reviewed. The most important effort is that, following similar activities in the West such as ECARP and AIAA Drag Prediction Workshops, a series of workshops on credible CFD simulations had been initiated. These workshops were with ambitions to assess the status of CFD in China. Another major effort is an ongoing project to establish a software platform for studying the credibility of CFD solvers and performing credible CFD simulations. The platform, named WiseCFD, was designed to implement a seamless CFD process and to circumvent tedious repeating manual operations. It had also been a powerful job manager for CFD with capabilities to support plug and play $(\mathrm{PnP})$ solver integration as well as distributed or parallel computations. Some future work on WiseCFD was proposed, and also envisioned was how WiseCFD and the European QNET-CFD Knowledge Base can benefit mutually.
\end{abstract}

\section{Introduction}

In the last decades, computational fluid dynamics (CFD) has undergone a strong development and has become a powerful tool both for the analysis and understanding of fluid dynamics phenomena, and for the design and optimization of aerodynamic performance of aircrafts or aerospace vehicles. This progress has been made possible with the advent, in the meantime, of faster and faster supercomputers with increasing memory capabilities, and with the rapid progress of modern numerical computing technology. However, the limitations of CFD need to be addressed due to the lack of knowledge of physical phenomena and appropriate physical modeling as well as limited experimental information. In addition, CFD is strongly affected by the numerical methodology employed (e.g., geometrical modeling, spatial discretization scheme, time accuracy computing, etc.) and computer resources. Therefore, the question of the credibility of the numerical solution is naturally proposed. In other words, to make the numerical simulations practicable, a crux stage is to perform a credibility analysis for the simulation, whose primary target is to assess and eliminate uncertainty in computation as possible. In terms of the authoritative guidance given by AIAA in 1998, the basic activities are CFD verification and validation $(\mathrm{V} \& \mathrm{~V})[1]$.
In developed countries, the activities related to CFD credibility analysis started early, and were paid extensive attentions, especially that they had laid many special projects and workshops with the intent of developing large databases of numerical solutions and experimental data of basic reference test cases to be used for CFD V\&V. The ECARP (European Computational Aerodynamics Research Project) involved 39 European partners for validation of CFD codes and assessment of turbulence models in Europe during the period 1993 to 1995 with a special research edition for close in 1997 [2]. At the end of 1998, in order to provide the scientific and industrial communities with a validation and experimental methodologies ranging from subsonic, transonic to supersonic, and hypersonic regimes, the European Community Commission began to support the setup of the Thematic Network FLOWNET (Flow Library on the Web network). The ultimate goal of this thematic network is to stimulate collaboration between industrial and research partners in order to evaluate continuously the quality of the simulations and the performance of CFD software, the scope being to improve complex design in aeronautical and aerospace industry [3]. In 2000, 44 participating organizations across Europe were brought together for a four-year project QNET-CFD, EU Network on Quality and Trust in the Industrial Application of Computational Fluid 
Dynamics [4]. Its main objective was to improve the level of trust that can be placed in industrial CFD calculations by assembling, structuring, and collating existing knowledge encapsulating the performance of models underlying the current generation of CFD codes. During the course of the project, QNET-CFD sponsored series of workshops in 2001, 2002, and 2003. Following the end of the EU Network in July 2004, its Knowledge Base passed to the control of a committee of the European Research Community of Flow, Turbulence, and Combustion (ERCOFTAC) with the remit of enhancing and expanding the Knowledge Base and bringing it online for the benefit of applied fluid dynamicists and CFD users worldwide.

AIAA Applied Aerodynamics Technical Committee (APATC) had sponsored a series of Drag Prediction Workshops since 2001. The objectives of these workshops were (1) to assess the state-of-the-art computational methods as practical aerodynamics tools for aircraft forces and moment prediction, (2) to impartially evaluate the effectiveness of existing computer codes and modeling techniques, and (3) to identify areas needing additional research and development. The first Drag Prediction Workshop (AIAA-DPW-I) was held in June of 2001. Its challenge was to compute the lift, drag, and pitching moment for the DLR-F4 subsonic wing-body transport configuration [5]. The second Drag Prediction Workshop (AIAA-DPW-II) was held in June of 2003. At this time the emphasis was on the determination of installed pylon-nacelle drag increments and on grid refinement studies with the hope of seeing reduced code-tocode scatter. The geometries used were DLR-F6 wing-body (WB) and wing-body-pylon-nacelle (WBPN) combinations [6]. The third Drag Prediction Workshop (AIAA-DPWIII) is planned to be held in June 2006. The focus of the workshop will be on "blind" drag prediction accuracy; a priori experimental data will not be available for comparison. In addition to the DLR-F6 wing-body with or without FX2B faring transport models, two wing-alone models, DPW-W1 and DPW-W2, are also included to encourage academic participation and allow more exhaustive grid convergence studies [7].

In March 2004, in order to more broadly assess the current capabilities of different computational methodologies, the CFD Validation of Synthetic Jet and Turbulence Separation Control (CFDVAL2004) workshop was held in Williamsburg, Virginia [8]. Three different test cases, all of which were carried out experimentally by NASA Langley Research Center, exercised various aspects related to the flow physics of separation control. The workshop was structured to the series of Refined Turbulence Modeling workshops sponsored by the ERCOFTAC, 10th of which was held in France in October 2002.

Comparatively, similar activities are rare in the past in China. In recent years the status is gradually changing. The theory and application studies for CFD credibility are paid much more attentions. Since 2000, CFD credibility analysis and experimental verification for aerodynamic numerical software had been determined a national key project among aerodynamic advanced research projects. From then on, many efforts for credible CFD simulations were made. In this paper our goal is to outline some outstanding efforts among them. The first important effort is The National CFD Uniform Test Cases Computation Workshop, following similar activities in the West such as ECARP and AIAA Drag Prediction Workshops. Such series of workshops were jointly sponsored by CARDC and ACTRI with intent to assess the state-of-the-art computational fluid dynamics (CFD) of China and to identify research areas in the near future. Another is WiseCFD software platform for aerodynamic credibility analysis, which is one of prominent outputs of an ongoing project at ACTRI [9]. It is designed to implement a seamless CFD process and to circumvent tedious repeating manual operations. Besides, efforts related to international collaborations for CFD credibility analysis are also reported; especially how WiseCFD and other validation databases, for example QNET-CFD, can benefit mutually is envisioned.

\section{China National CFD V\&V Workshops}

The mainstream collective CFD V\&V activities in China are two successive workshops organized by CARDC and ACTRI in 2003 and 2005, respectively. Besides, some following actions are also taken later on [10]. Both of these workshops are to assess the state of the art of CFD in China, and to identify areas needing additional research and development to promote prediction accuracy.

The first effort to hold a workshop in 2003 was abandoned for some reasons, but resulted in a great promotion of relative researches and a special edition of papers [11]. The special edition was composed of 17 papers from 9 organizations. The selected test cases include the DLR-F4 wing-body combination, NLR-7301 two elements airfoil at high lift, and blunt cone at hypersonic speed. The emphasis is the prediction accuracy for drag and heat flux. The participators are required to perform their computations using the geometry and grid files provided by head organizers and are also encouraged to generate their own grid for simulation. The geometry of DLR-F4 configuration is the same as that of AIAA DPW-I [5]. Its challenge for this workshop is to compute the lift, drag, and pressure distribution at typical wing section $0.185,0.238,0.331,0.409,0.512,0.636$, and 0.844 along the wing for a specific condition $\left(\operatorname{Re}=3 \times 10^{6}\right)$ :

$$
M_{\infty}=0.75, \quad \alpha=0.93^{\circ}\left(C_{L}=0.6\right) .
$$

The dominant computational grid is provided by CARDC. Some of participators also generate their own grid. There are 9 papers reporting 19 computational results. Only 2 results are obtained using Euler computation. The rest employ Reynolds-Averaged Navier-Stokes (RANS) technique with proper turbulent models. The typical turbulent models include Spalart-Allmaras (SA) and Baldwin-Lomax (BL). A statistical analysis for these results is shown in Figure 1. It is observed that results from the workshop are quite scattered. Most of the results have over-predicted the lift and drag. For the case of NLR-7301 high-lift airfoil, two geometries are considered depending upon the gap size between the wing and the single-slotted trailing edge flag, respectively, $1.3 \%$ 


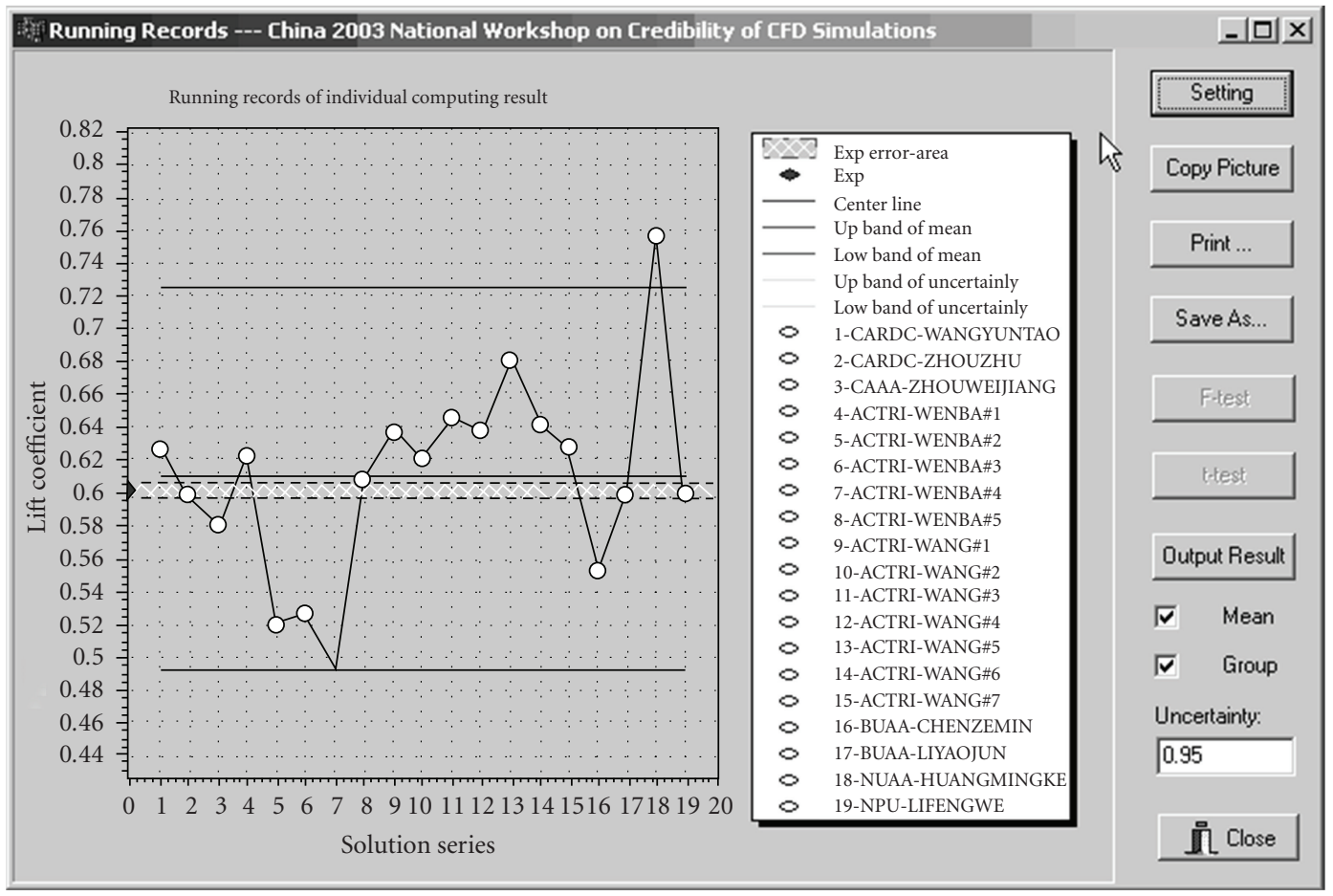

(a) Lift coefficient

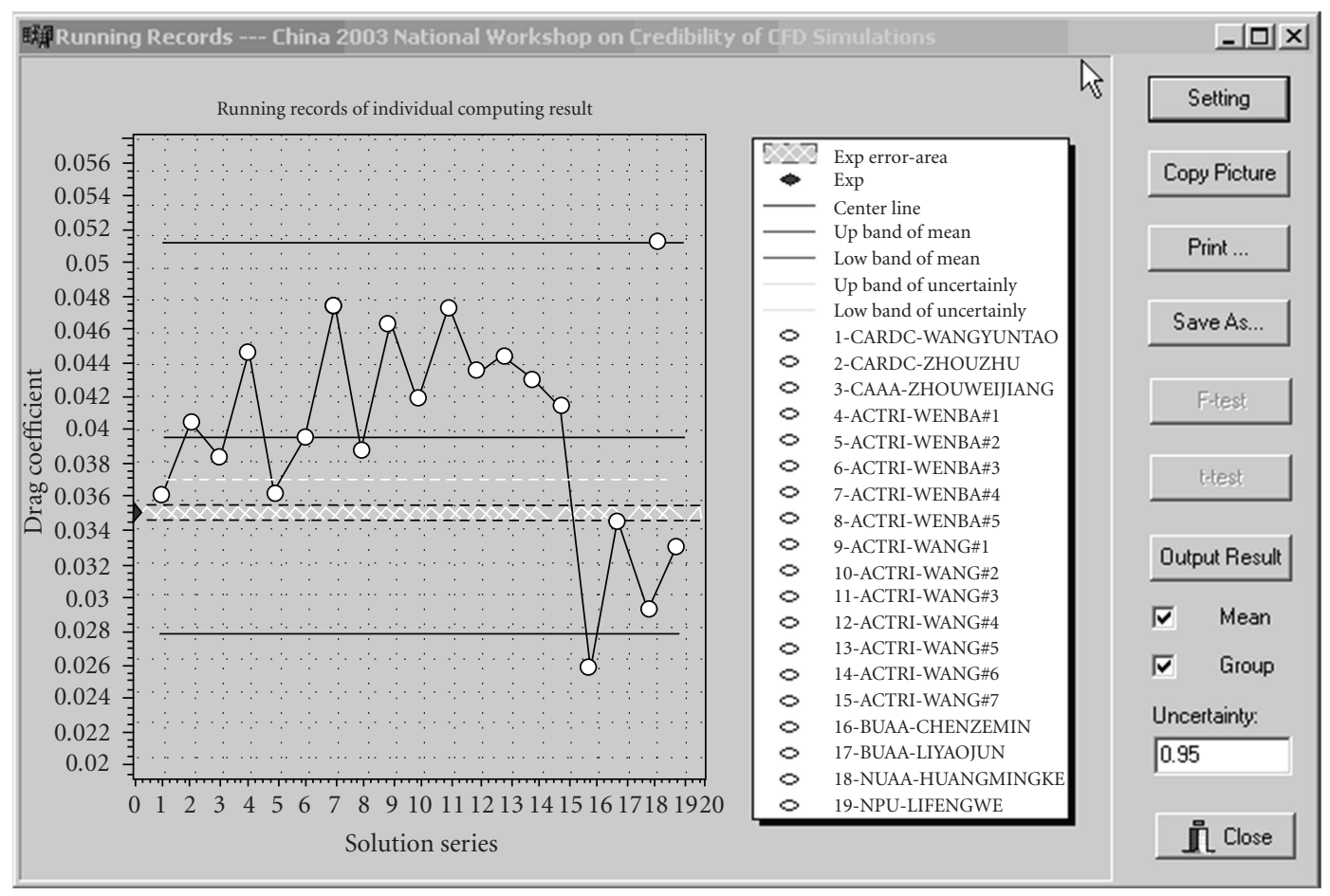

(b) Drag coefficient

FIgure 1: Statistical analysis of computational results for DLR-F4 wing-body configuration from the 1st National CFD Uniform Test Cases Computation Workshop. 


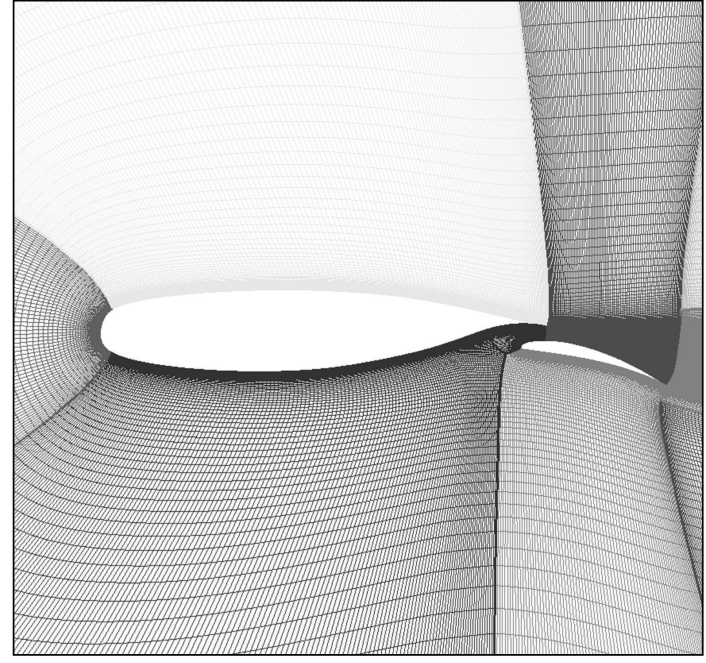

Figure 2: NLR-7301 two elements high lift airfoil and its typical grid topology from ECARP and FLOWNET (2.6\% flap-gap).

and $2.6 \%$, being as a percentage value of the main wing chord. The computational conditions are

(1) $1.3 \%$ flap-gap

$M_{\infty}=0.185, \quad \alpha=6^{\circ}, \quad \operatorname{Re}=2.51 \times 10^{6}$ and

(2) $2.6 \%$ flap-gap

$M_{\infty}=0.185, \quad \alpha=13.1^{\circ}, \quad \operatorname{Re}=2.51 \times 10^{6}$.

The dominant grids used in this workshop for such configurations are multi-block structured patched ones, which can be also found from ECARP and FLOWNET libraries. Their topology and cells number are completely identical with 9 blocks and 144832 cells. In Figure 2, a typical grid topology is shown. Results required to be submitted include

(1) lift coefficient, drag coefficient, and their convergent histories,

(2) pressure coefficient distributions of the main wing and the flap,

(3) skin friction coefficient distributions of the main wing and the flap, and

(4) velocity profiles of the boundary layer at typical locations.

There are 5 contributors for the test case. Among them, besides results of RANS plus turbulent models, some results of Large Eddy Simulation (LES) are also reported. Figure 3 shows a typical pressure coefficient distribution computational result by ACTRI employing RANS with SA turbulent model. For this challenge, in order to promote the prediction accuracy for large flow separation near the wall, a wider and more elaborate variety of turbulent models (SA, BL, $k-\omega$, Jones-Launder, JB, and SGS, etc.) have been considered in these contributions. Same scatters are found for this test case.

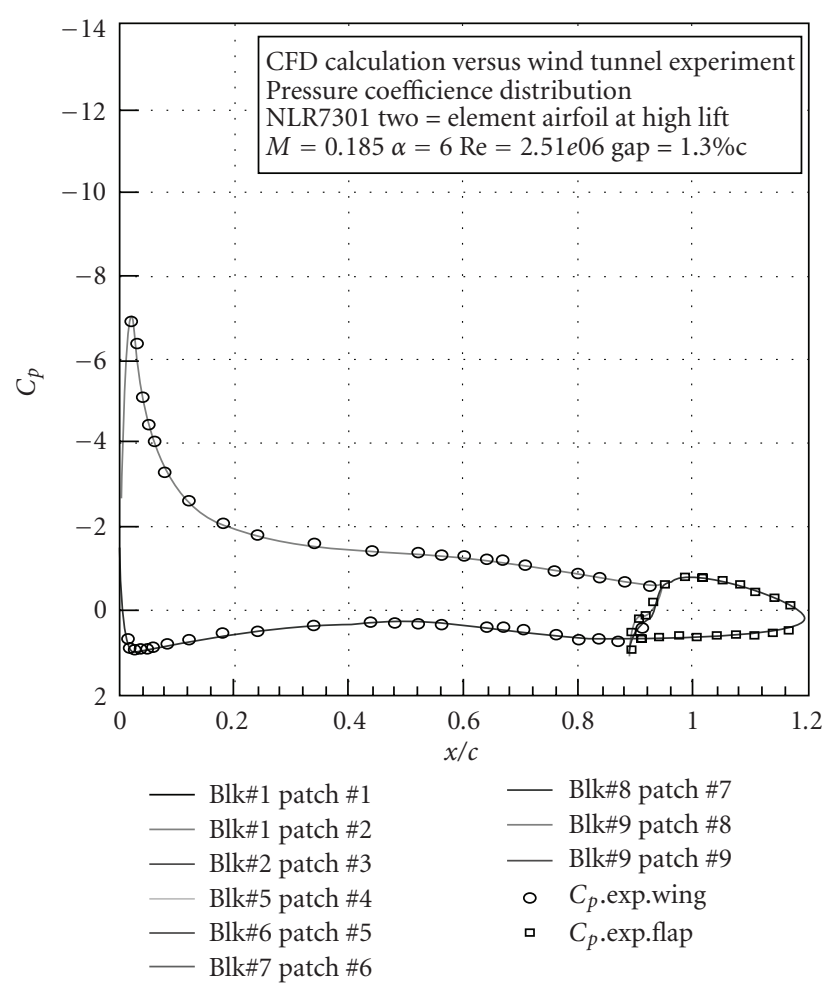

FIGURE 3: Pressure coefficient distribution by ACTRI for NLR-7301 two elements airfoil (1.3\% flap-gap).

The geometry and its dominant computational grid of the third test case, hypersonic blunt cone, are both provided by CARDC. Its challenge is to accurately predict heat flux for the following conditions:

$$
\begin{gathered}
M_{\infty}=10.6, \quad T_{\infty}=47.3 \mathrm{~K}, \quad T_{\text {wall }}=294.44 \mathrm{~K}, \\
\alpha=0^{\circ}, 20^{\circ}, \quad \operatorname{Re}=3.937 \times 10^{6} .
\end{gathered}
$$

There are 6 contributors having reported their results on the workshop. Figure 4 shows a typical result for this test case by CARDC. From these contributions, one important observation is that a higher order scheme is obviously superior for heart flux prediction based on the same computational grid.

The second national effort for credible CFD simulations started from September 2004. The formal workshop was held in Inner Mongolia, June 2005. The emphasis of this workshop is the simulation of high attack angle problem, where the CT-1 standard model provided by CARDC [12] is computed for six main force coefficients (i.e., axial force coefficient, normal force coefficient, side force coefficient, $X$ force coefficient, $Y$ force coefficient, and $Z$ force coefficient), lift coefficient, and drag coefficient. The computations were performed at the following flow conditions: $M_{\infty}=0.5, \mathrm{Re}=$ $1.4 \times 10^{6}$, angles of attack between $-5^{\circ}$, and $105^{\circ}$, and sideslip angles $\beta=0^{\circ}, 5^{\circ}, 15^{\circ}$.

There are 34 attendees from 12 workgroups of 9 organizations. Most results are obtained by RANS technique plus proper turbulent models. The turbulent models involved include SA, BL, $k-\omega$, and SST. Part results are from 


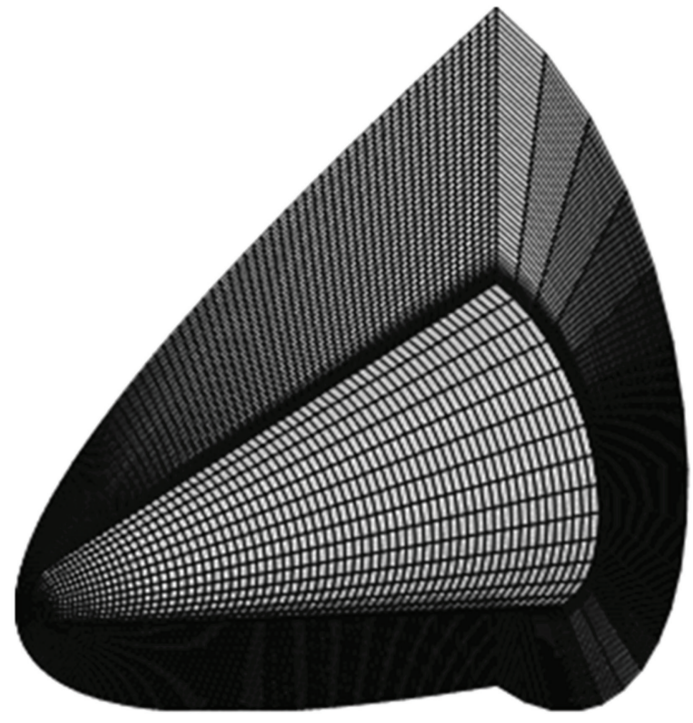

(a) Computational grid and topology

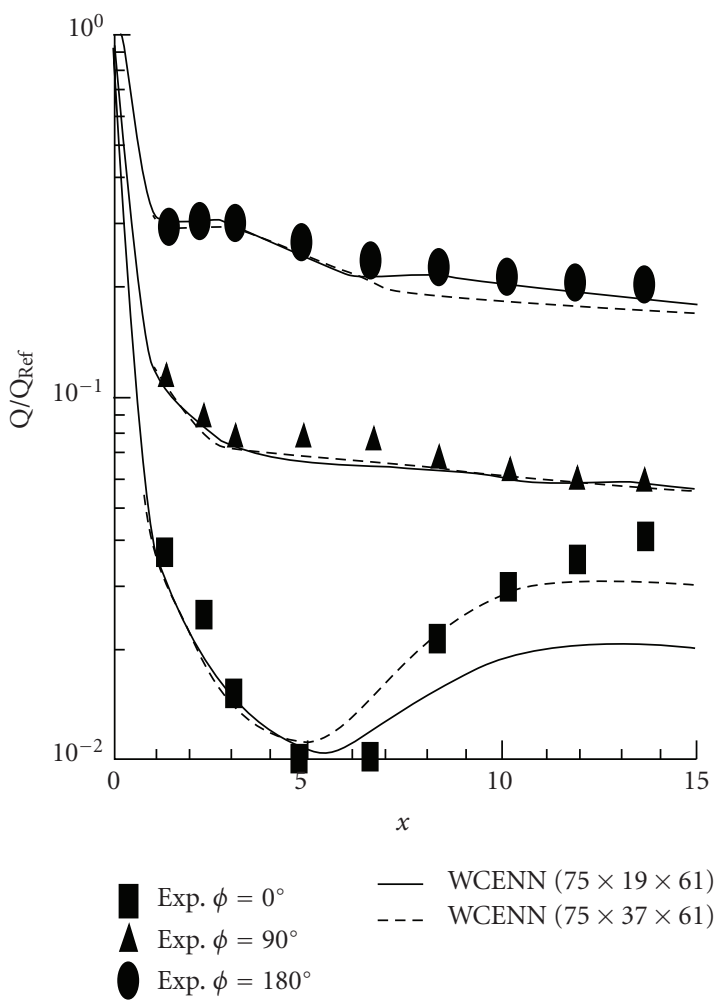

(b) Heat flux

FIgUre 4: Typical computational results for hypersonic blunt cone configuration from the 1st National CFD V\&V Workshop.

Euler computation. Besides, few results from Detached Eddy Simulation (DES, hybrids of LES and RANS) and Lattice Boltzmann Method (LBM) are reported. Figure 5 shows a typical result from the workshop, where the lift coefficients from different contributors are given. Results of this workshop exhibit the state of the art of CFD researches in China.

It is shown that for a high attack angle problem, great progresses have been made in the recent years. Briefly speaking, for CT-1 model, we had concluded that (1) the computational aerodynamic characteristic (e.g., lift, drag, and pitching moment) under medium attack angle is of good agreement with experimental data, (2) the computational angle to occur unsymmetrical side force agrees with experimental data well, while the amplitude of the side force is closed to from experiments, and (3) when the sideslip angle does not equal to zero, the computational angle making the yawing moment in reverse agrees with experimental data by and large. Nevertheless, some limits are also observed. They are (1) the attack angle corresponding to the maximal lift coefficient from computations is offset to that from experiments, (2) there exist obvious gaps between computations and experiments for results at attack angles between $40^{\circ}$ and $60^{\circ}$, and (3) prediction accuracy of moment characteristic at high attack angle is not enough.

\section{Integrated Software Platform for Credible CFD Simulations: WiseCFD}

Since 2000, ACTRI had begun to develop an integrated software platform for aid to assess the credibility of CFD solvers and to perform a credible CFD simulation with less human errors. Its main objective is to develop an intuitive user environment that allows the user to prepare, submit, monitor, and manage large numbers of CFD simulation jobs for parametric studies during the course to perform CFD credibility analysis, and also to implement an integrated management for data and software.

The system architecture of the WiseCFD platform is shown in Figure 6, which comprises a V\&V Case Library and five major functional modules as its main parts. The Case Library can be alternatively regarded as a special database for CFD verification and validation. Large amount of reliable data had been collated in the past several years and integrated in the database. The five functional modules are used to implement different functions in the system, for example, solver management, case data management, parameter sensitivity management, the batched job management, and data analysis and comparison. In this way, an automatic seamless CFD process is built up, which thus greatly enhances computing efficiency and electively reduces uncertainty in simulation.

Currently, the WiseCFD platform has the published version 2.0, whose main graphic user interface (GUI) is shown in Figure 7. There are four different view areas on the GUI that include (1) the toolbar area at the top, (2) the case catalog tree area at the left, (3) the operating panel area in the middle, and (4) the information hint area at the bottom. The toolbar is used to provide a shortcut access to different functions related to software management, computing job management, data backup, or system help. The view area for case catalog tree is used to display hierarchy-arranged case data in a way similar to Windows catalog tree. The top level 


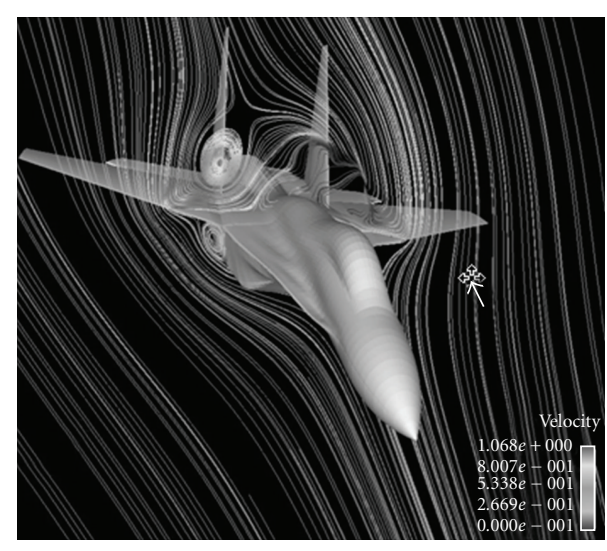

(a) CT-1 Standard Aircraft Model at High AoA

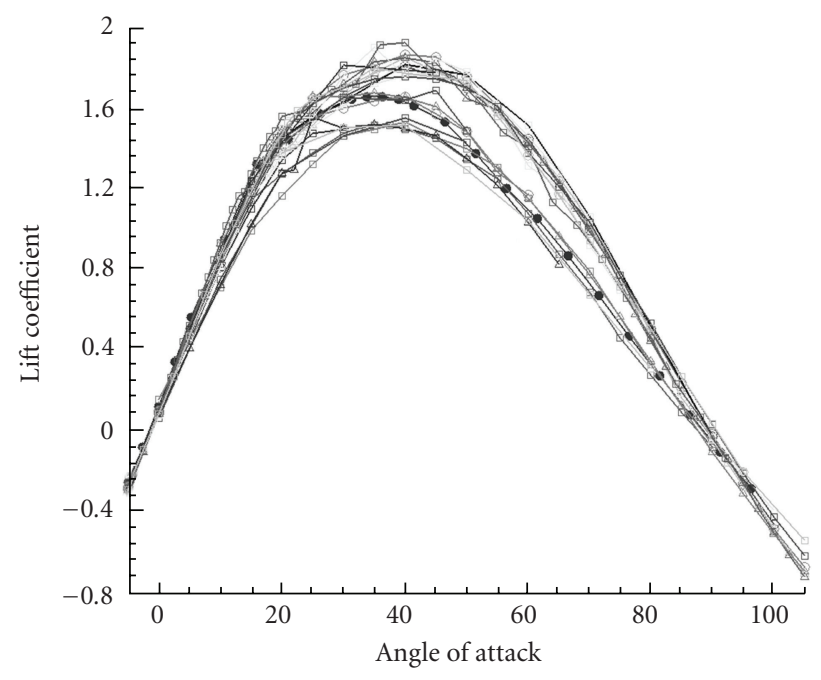

$\rightarrow$ Wind tunnel experimental data
$\because$ CARDC-ZhouZhu
$\because$ CARDC-ChenXiaoDong
- CARDC-WangGuangXue
- CARDC-XiaoHanShan-Euler
$\square$ CAAA-ZhouWeijiang
$\square$ BUAA-YanChao
$\square$ SSC-ZhangSaiJin
$\square$ NPU-LiFengWei-Euler
- NPU-LiFengWei-RANS
ACTRI-WiseMan-ProvidedGrid

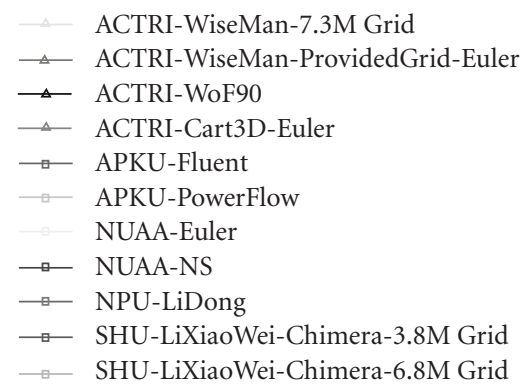

(b) lift coefficients

Figure 5: A typical computational result for high AoA model from the 2nd National CFD Uniform Test Cases Computation Workshop.

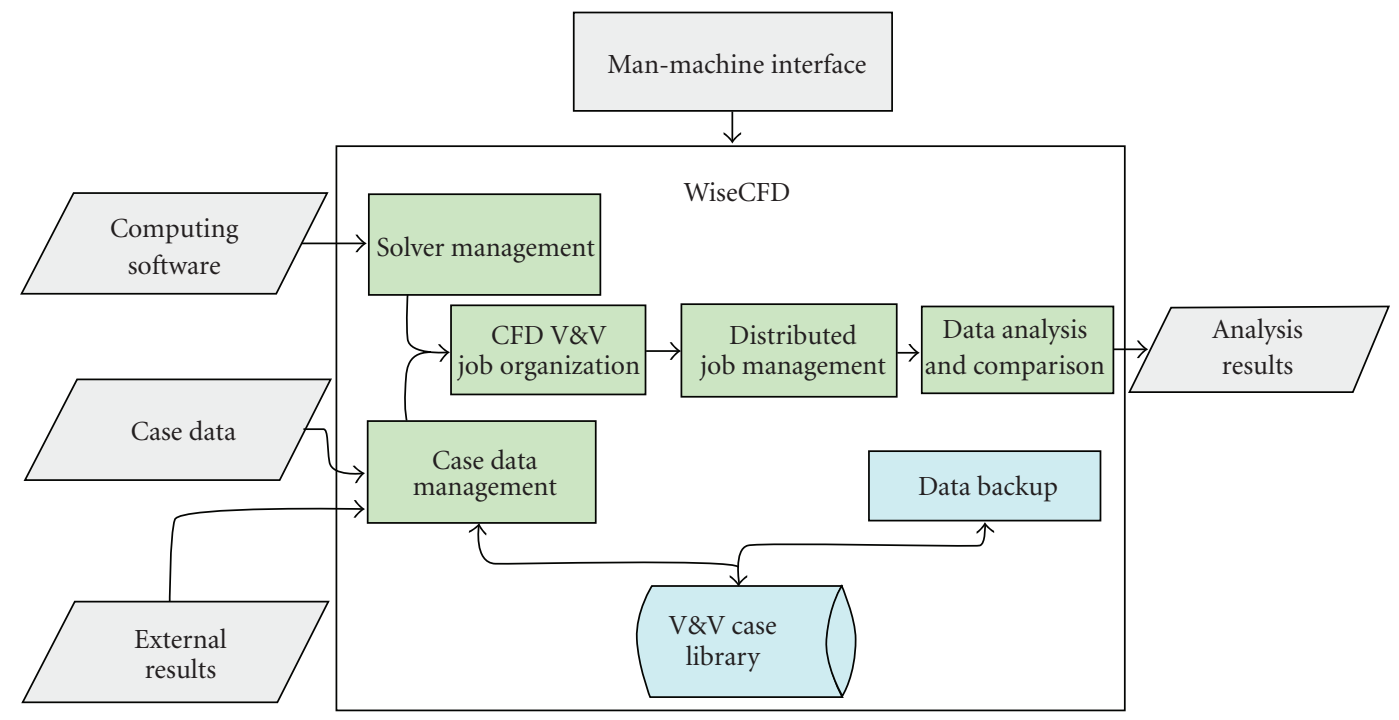

FIGURE 6: System architecture of the WiseCFD platform.

is the case type, which can be designated as three different classes: verification case, validation case, and application challenge case. The second level is the practical test case. The third level is the computational solver to be used for the test case, and the fourth is the so-called study case that is used to record practical analysis activity. At each level, different operation panels will be shown in the panel view area. Through these panels, the functions related to case data management, computing software management, study case management, computational results management, and data 


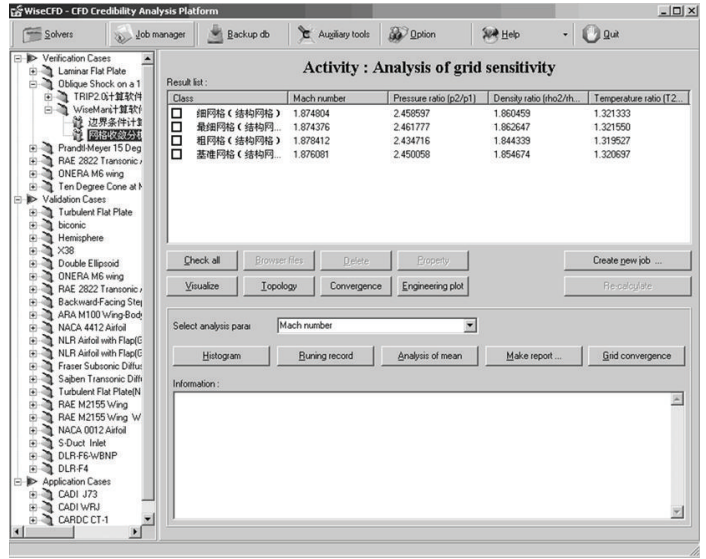

FIgUre 7: Graphic user interface (GUI) of the WiseCFD Platform.

analysis and comparison can be accessed. The main features of WiseCFD v2.0 are briefly summarized here:

(1) it provides a convenient and user-friendly graphical user interface;

(2) it provides an extensible database for CFD verification and validation, in which 6 verification cases, 20 validation cases and 3 application challenge cases are available in current version;

(3) with an extensible open framework for software integration, and with the use of data-exchange standards (e.g., plot3d and CGNS), it supports the plug and play $(\mathrm{PnP})$ software integration, from which different in-house and commercial CFD codes have been integrated in this simple way;

(4) with the function for parameter sensitivity analysis and management, it allows the user to prepare large amount of different input files for CFD credibility analysis in similar simple way;

(5) with the capability for distributed job management, it allows the user easily to submit and monitor the large batched CFD jobs, and to effectively utilize available computing resources under the Windows and the Linux cluster environments as well;

(6) with abundant auxiliary tools for data analysis and comparisons, it not only provides a simple way for aerodynamic data extraction (i.e., WiseADC), engineering curve plot (i.e., WiseECG), data statistical analysis (i.e., WiseSAT), and grid convergence analysis (i.e., WiseGCI), but also customizes manifold interfaces in script with general commercial pre- and postprocessors such as ICEM-CFD, Gridgen, Gambi, Tecplot, Ensight, and Visit;

(7) it has a perfect help system, in which not only the help documents related to system operations are available, but also a collection of literatures related to CFD credibility analysis is provided; the collection has collected and collated many important literatures that almost cover each process in CFD verification, validation, prediction, and calibration.

\section{Future Work and Conclusions}

In the present paper, we have outlined some recent efforts for CFD credible simulations in China, whose basic activities are verification and validation in terms of international authority. The aspects of verification and validation must always be addressed with an emphasis on the quantification of the uncertainties due to the model assumptions (either physical or geometrical) and to the numerical and experimental approximations. There are various roadmaps for CFD V\&V, but the most regular one is through different workshops. Following similar activities in the West such as ECARP and Drag Prediction Workshops, two successive national workshops for V\&V of CFD simulations had been organized by CARDC and ACTRI in the past years with intent to assess the state of the art of computational fluid dynamics of China and to identify areas needing additional research and development. And most recently, some following actions have been initiated.

Development of a software platform for performing credibility analysis is a creative idea. The attempt has been tried in ACTRI to develop the WiseCFD platform. The main components of the platform include the Case Library, the graphical user interface for jobs management, the tool for credibility and parameter analysis, and the auxiliary tools for data exaction, analysis, and comparisons, where the Case Library for CFD verification and validation is the kernel for the system. Large amount of experimental data and CFD results have been integrated, which results in a useful validation database similar to that of FLOWNET and QNET-CFD in Europe. Nowadays the WiseCFD platform has integrated several in-house and commercial RANS CFD codes using its $\mathrm{PnP}$ integrated strategy. In the near future, it is hoped to involve some more CFD codes with advanced numerical methods such as DES or even LES/DNS for better prediction of flow structures around, for example an aircraft, and also provide the capabilities to perform credibility analysis for such type of solvers.

However, CFD V\&V requires much involvement. It's still a long way for China. China should be more involved in international activities. Through these collaborations, it is expected that the West and China can benefit mutually. For example, in connection with the QNET-CFD thematic network, WiseCFD can contribute and enjoy available resources.

\section{Acknowledgments}

This work is supported by the State Key Development Program of Basic Research of China (973) under Grant no. 2009CB723804. The authors would also like to express their acknowledgment for anonymous reviewers for their good comments to enrich this work.

\section{References}

[1] AIAA-G-077-1998, Guide for the Verification and Validation of Computational Fluid Dynamics Simulations, American Institute of Aeronautics and Astronautics, 1998.

[2] W. Haase, E. Chaput, E. Elsholz, M. A. Leschziner, and U. R. Muller, "ECARP-European computational aerodynamics 
research project: validation of CFD codes and assessment of turbulent models," Notes on Numerical Fluid Mechanics, vol. 58, 1997.

[3] M. Marini, R. Paoli, F. Grasso, J. Periaux, and J. A. Desideri, "Verification and validation in computational fluid dynamics: the FLOWnet database experience," JSME International Journal, Series B, vol. 45, no. 1, pp. 15-21, 2002.

[4] C. Hirsch, "The QNET-CFD project," The QNET-CFD Network Newsletter, vol. 2, no. 4, pp. 4-5, 2004.

[5] D. W. Levy, T. Zickuhr, J. Vassberg et al., "Summary of Data from the first AIAA CFD drag prediction workshop," AIAA paper 2002-0841, 2002.

[6] M. J. Hemsch and J. H. Morrison, "Statistical analysis of CFD solutions from 2nd Drag prediction workshop," AIAA paper 2004-0556, 2004.

[7] http://aaac.larc.nasa.gov/tsab/cfdlarc/aiaa-dpw/.

[8] C. L. Rumsey, T. B. Gatski, W. L. Sellers 3rd, V. N. Vatsa, and S. A. Viken, "Summary of the 2004 CFD validation workshop on synthetic jets and turbulent separation control," AIAA Paper 2004-2217, 2004.

[9] Y. H. Liang, Y. Yang, Z. Zhu et al., "CFD credibility analysis platform WiseCFD," in Proceeding of 12th National Computational Fluid Dynamics Conference, pp. 775-780, Chinese Aeronautics Computation Technique Research Institute, Xian, China, 2004.

[10] W. Bai, "Remark on CFD application techniques for aerodynamic design of large civil aircraft," in The Application and Development of CFD in Large Civil Aircraft, G. H. Wu and Y. C. Chen, Eds., 2009.

[11] Z. B. Chen and W. Bai, "Special edition for credible Aerodynamic computations," Aeronautical Computer Technique, vol. 32, 2004 (Chinese).

[12] J. Q. Wu, Z. L. Fan, Z. He, and Y. J. Wang, "Research on the tests technology at high angles of attack in $2.4 \mathrm{~m}$ transonic wind tunnel," Experiments and Measurements in Fluid Mechanics, vol. 18, no. 4, pp. 43-48, 2004 (Chinese). 

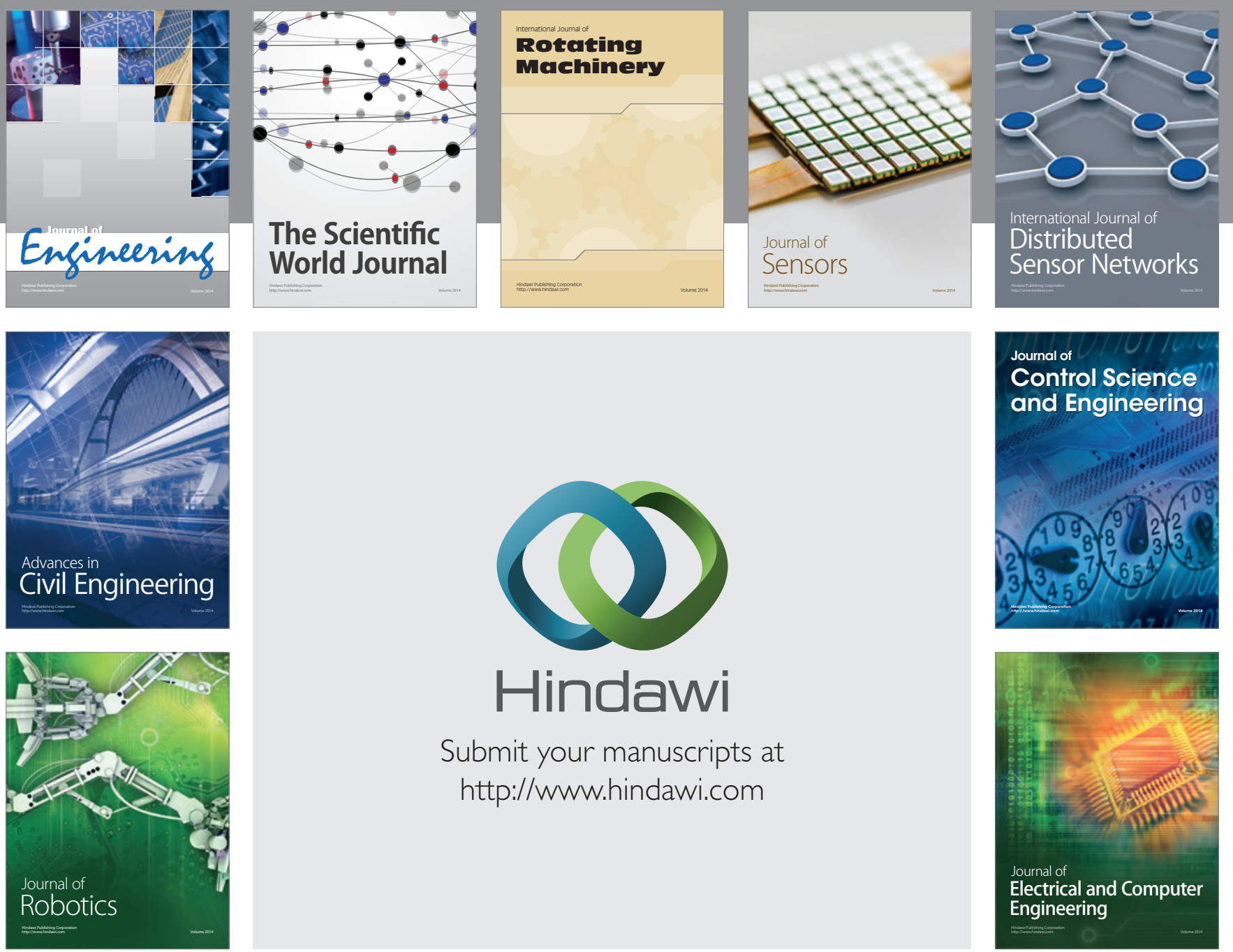

Submit your manuscripts at

http://www.hindawi.com
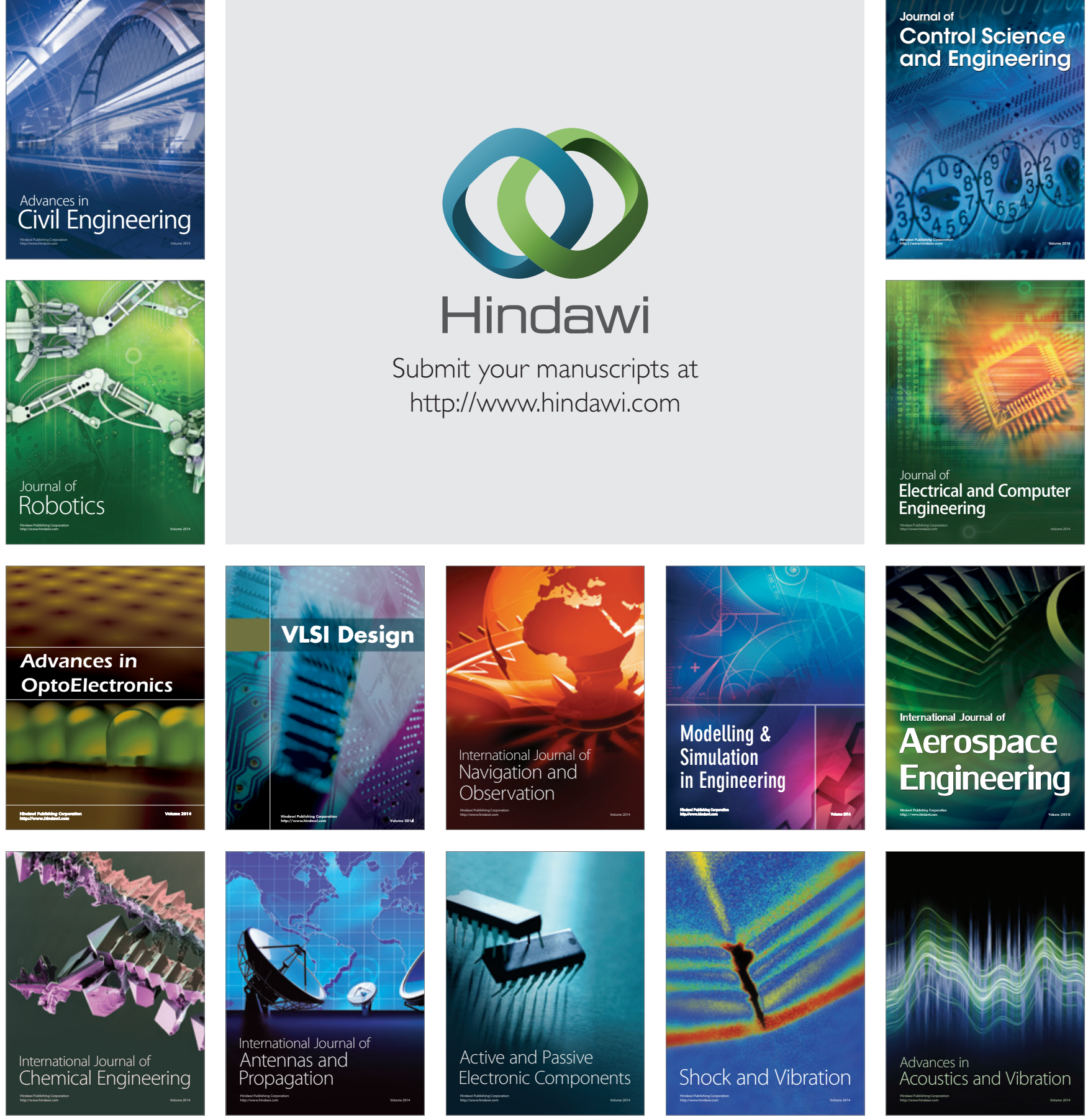